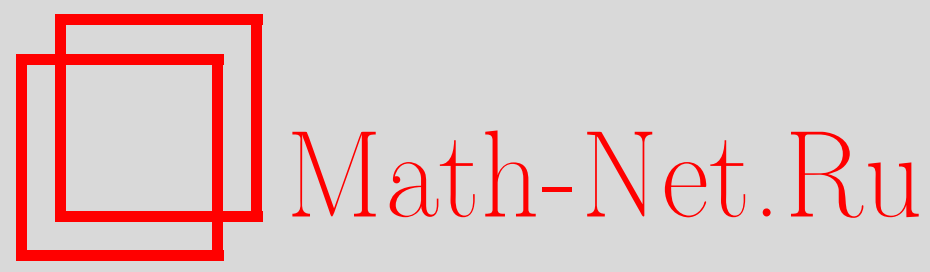

А. С. Белов, Замечания о сходимости (ограниченности) в среднем частных сумм тригонометрического ряда, $\mathrm{Ma-}$ тем. заметки, 2002, том 71, выпуск 6, 807-817

DOI: https://doi.org/10.4213/mzm386

Использование Общероссийского математического портала Math-Net.Ru подразумевает, что вы прочитали и согласны с пользовательским соглашением http://www.mathnet.ru/rus/agreement

Параметры загрузки:

IP : 3.85 .183 .62

26 апреля 2023 г., 13:18:56

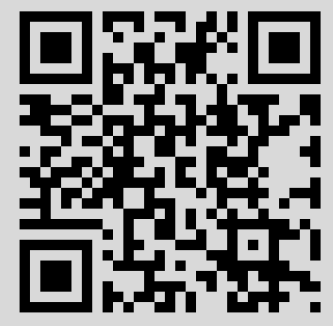


УДК 517.5

\section{ЗАМЕЧАНИЯ О СХОДИМОСТИ (ОГРАНИЧЕННОСТИ) В СРЕДНЕМ ЧАСТНЫХ СУММ ТРИГОНОМЕТРИЧЕСКОГО РЯДА}

\section{А. С. Белов}

В статье даются довольно общие условия на коэффициенты $\left\{a_{n}\right\}_{n=1}^{\infty}$ как четного, так и нечетного тригонометрического ряда Фурье, при выполнении которых верно утверждение: частные суммы ряда сходятся (соответственно ограничены) в метрике $L$ тогда и только тогда, когда $\sum_{k=[n / 2]}^{2 n}\left|a_{k}\right| /(|n-k|+1)=o(1)$ (соответственно $=O(1))$.

Библиограбия: 8 названий.

Пусть

$$
\sum_{n=-\infty}^{\infty} c_{n} e^{i n x}=\frac{1}{2} a_{0}+\sum_{n=1}^{\infty}\left(a_{n} \cos (n x)+b_{n} \sin (n x)\right)
$$

- произвольный тригонометрический ряд, записанный в действительной или комплексной форме. Далее используются обычные обозначения:

$$
\begin{gathered}
a_{n}=c_{n}+c_{-n}, \quad b_{n}=\left(c_{n}-c_{-n}\right) i, \\
A_{n}(x)=c_{n} e^{i n x}+c_{-n} e^{-i n x}=a_{n} \cos (n x)+b_{n} \sin (n x), \\
S_{n}(x)=c_{0}+\sum_{k=1}^{n} A_{k}(x), \quad \widetilde{S}_{n}(x)=-i \sum_{k=-n}^{n} \operatorname{sign}(k) c_{k} e^{i k x}, \\
\sigma_{n}(x)=\frac{1}{n+1} \sum_{k=0}^{n} S_{k}(x)=S_{n}(x)-\frac{1}{n+1} \sum_{k=1}^{n} k A_{k}(x) \quad \text { при всех } n \geqslant 0 .
\end{gathered}
$$

Если $f \in L_{2 \pi}$, то

$$
\|f\|=\|f\|_{1}=\|f\|_{L}=\frac{1}{2 \pi} \int_{0}^{2 \pi}|f(x)| d x .
$$

Квадратные скобки, как обычно, обозначают целую часть числа, а черта над числом - переход к комплексно-сопряженному числу.

Последовательность комплексных чисел $\left\{c_{n}\right\}_{n=-\infty}^{+\infty}$ назьвается LC-nоследовательностью (соответственно LB-последовательностью), если

$$
\left\|\sigma_{n}-S_{n}\right\|=o(1) \quad(\text { соответственно }=O(1)) .
$$

Работа выполнена при финансовой поддержке Российского фонда фундаментальных исследований, грант № 99-01-00062, и фонда INTAS, грант № 99-01080. 
Кратко это будем записывать в виде

$$
\left\{c_{n}\right\}_{n=-\infty}^{+\infty} \in \mathrm{LC} \quad(\text { соответственно } \in \mathrm{LB})
$$

В (2) и во всех аналогичных соотношениях далее, конечно, предполагается, что $n$ стремится к бесконечности.

Эта статья посвящена изучению условий на коэффищиенты тригонометрического ряда (1), при которых верно соотношение (2). По определению условия (2) и (3) означают одно и то же. Поскольку понятие LC-последовательности (соответственно LB-последовательности) играет важную роль в дальнейшем изложении, то мы сначала более подробно остановимся на этом понятии, а затем изложим полученные новые результаты и их отношение к известньм теоремам.

Если $\left\{c_{n}\right\}_{n=-\infty}^{+\infty}$ и $\left\{c_{n}^{\prime}\right\}_{n=-\infty}^{+\infty}$ являются LC-последовательностями (соответственно LB-последовательностями), то, очевидно, для любых комплексных чисел $\alpha$ и $\alpha^{\prime}$ последовательность $\left\{\alpha c_{n}+\alpha^{\prime} c_{n}^{\prime}\right\} \in \mathrm{LC}$ (соответственно $\left.\in \mathrm{LB}\right)$, т.е. совокупность LC-последовательностей (соответственно LB-последовательностей) образует линейное комплексное пространство (см. также замечание 1 в конце статьи).

Известно (см. [1, лемма 3] или [2, леммы 1 и 2]), что условие (2) эквивалентно каждому из следующих условий:

$$
\begin{gathered}
\left\|\widetilde{\sigma}_{n}-\widetilde{S}_{n}\right\|=o(1) \quad(\text { соответственно }=O(1)) \\
\max _{m=n, \ldots, 2 n-1}\left\|S_{m}-S_{n-1}\right\|=o(1) \quad(\text { соответственно }=O(1)), \\
\max _{m=n, \ldots, 2 n-1}\left(\left\|\sum_{k=n}^{m} c_{-k} e^{-i k x}\right\|+\left\|\sum_{k=n}^{m} c_{k} e^{i k x}\right\|\right)=o(1) \quad(\text { соответственно }=O(1)) .
\end{gathered}
$$

Таким образом, каждое из условий (4)-(6) эквивалентно условию (3).

Отметим, что для любой $f \in L_{2 \pi}$ нормы функций $f(x), \bar{f}(x)$ и $f(-x)$ всегда совпадают. Поэтому если верно (3), то в силу (4) последовательности $\left\{-i \operatorname{sign}(n) c_{n}\right\}_{n=-\infty}^{+\infty}$, $\left\{\bar{c}_{n}\right\}_{n=-\infty}^{+\infty}$ и $\left\{c_{-n}\right\}_{n=-\infty}^{+\infty}$ также являются LC-последовательностями (соответственно LВ-последовательностями).

Будем писать, что последовательность $\left\{c_{n}\right\}_{n=m}^{\infty} \in \mathrm{LC}$ (соответственно $\in \mathrm{LB}$ ), если, полагая $c_{n}=0$ при всех $n<m$, получим последовательность, удовлетворяющую условию (3). Аналогично трактуется запись $\left\{c_{n}\right\}_{n=-\infty}^{m} \in \mathrm{LC}$ (соответственно $\in \mathrm{LB}$ ). Поэтому (3) эквивалентно условию, что последовательности

$$
\left\{c_{n}\right\}_{n=1}^{\infty},\left\{c_{-n}\right\}_{n=1}^{\infty} \in \mathrm{LC} \quad(\text { соответственно } \in \mathrm{LB}),
$$

а последнее эквивалентно условию, что последовательности

$\left\{\operatorname{Re} c_{n}\right\}_{n=1}^{\infty},\left\{\operatorname{Im} c_{n}\right\}_{n=1}^{\infty},\left\{\operatorname{Re} c_{-n}\right\}_{n=1}^{\infty},\left\{\operatorname{Im} c_{-n}\right\}_{n=1}^{\infty} \in \mathrm{LC} \quad$ (соответственно $\left.\in \mathrm{LB}\right)$

Отметим также, что если ряд (1) задан в действительной форме, то условие (7) эквивалентно условию

$$
\left\{a_{n}\right\}_{n=1}^{\infty},\left\{b_{n}\right\}_{n=1}^{\infty} \in \mathrm{LC} \quad(\text { соответственно } \in \mathrm{LB})
$$


а это последнее эквивалентно условию

$$
\left.\left\{\operatorname{Re} a_{n}\right\}_{n=1}^{\infty},\left\{\operatorname{Re} b_{n}\right\}_{n=1}^{\infty},\left\{\operatorname{Im} a_{n}\right\}_{n=1}^{\infty},\left\{\operatorname{Im} b_{n}\right\}_{n=1}^{\infty} \in \mathrm{LC} \quad \text { (соответственно } \in \mathrm{LB}\right) .
$$

Таким образом, условия (2)-(9) эквивалентны между собой. В частности, для четного тригонометрического ряда

$$
\frac{1}{2} a_{0}+\sum_{n=1}^{\infty} a_{n} \cos (n x), \quad A_{n}(x)=a_{n} \cos (n x) \text { при } n \geqslant 0,
$$

с произвольными комплексными коэффициентами, соотношение (2) выполнено тогда и только тогда, когда

$$
\left\{a_{n}\right\}_{n=1}^{\infty} \in \mathrm{LC} \quad(\text { соответственно } \in \mathrm{LB}) .
$$

Аналогично, для нечетного тригонометрического ряда

$$
\sum_{n=1}^{\infty} a_{n} \sin (n x), \quad A_{n}(x)=a_{n} \sin (n x) \text { при } n \geqslant 0
$$

соотношение (2), где, конечно, частные суммы и суммы Фейера берутся для ряда (12), выполнено тогда и только тогда, когда верно (11). Поскольку (3) эквивалентно (6), то условие (11) эквивалентно условию

$$
\max _{m=n, \ldots, 2 n-1}\left\|\sum_{k=n}^{m} a_{k} e^{i k x}\right\|=o(1) \quad(\text { соответственно }=O(1)) .
$$

Из (13) вытекает, что если последовательность удовлетворяет условию (11), то $\left|a_{n}\right|=$ $o(1)($ соответственно $=O(1))$. Поэтому условие $(11)$ эквивалентно условию

$$
\left\{a_{n+1}\right\}_{n=1}^{\infty} \in \mathrm{LC} \quad(\text { соответственно } \in \mathrm{LB}) \text {. }
$$

Отсюда и из эквивалентности условий (3) и (7) следует, что для любого целого $s$ условие (3) эквивалентно условию

$$
\left\{c_{n+s}\right\}_{n=-\infty}^{+\infty} \in \mathrm{LC} \quad(\text { соответственно } \in \mathrm{LB}) \text {. }
$$

Некоторые другие свойства LC- и LB-последовательностей изложены далее в замечании 1.

Эта статья посвящена изучению некоторых LC- и LB-последовательностей специального вида. Отметим (см. [1, лемма 3$])$, что условие (13), а значит и (11), эквивалентно условию

$$
\max _{m=2^{n}, \ldots, 2^{n+1}}\left\|\sum_{k=2^{n}+1}^{m} a_{k} e^{i k x}\right\|=o(1) \quad(\text { соответственно }=O(1)) .
$$

Таким образом, удовлетворяющую условию (11) последовательность можно представлять себе сконструированной из наборов вида $\left\{a_{k}: k=2^{n}+1, \ldots, 2^{n+1}\right\}$ так, что выполнено (14).

В статье [2] доказана следующая 
ТЕоремА А. Если последовательность $\left\{a_{n}\right\}_{n=0}^{\infty}$ является LC-последовательностью (соответственно LВ-последовательностью), то выполнено условие

$$
\sum_{k=[n / 2]}^{2 n} \frac{\left|a_{k}\right|}{|k-n|+1}=o(1) \quad(\text { соответственно }=O(1)) \text {. }
$$

Подчеркнем, что во всех утверждениях этой статьи переход к случаю, указанному в кругльх скобках, производится во всех условиях одновременно.

Цель этой статьи - показать, что для некоторых достаточно общих последовательностей $\left\{a_{n}\right\}_{n=1}^{\infty}$ специального вида теорему А можно обратить.

Последовательность целых чисел

$$
\left\{N_{n}\right\}_{n=1}^{\infty}, \quad 0<N_{1}<N_{2}<\cdots
$$

называется конечно-лакунарной, если существует натуральное число $p$ такое, что

$$
\lambda=\inf _{n \geqslant 1} \frac{N_{n+p}}{N_{n}}>1 .
$$

Для случая $p=1$ последовательность (16) называется лакунарной (по Адамару). Из (17) следует, что при каждом натуральном $m$ будет верна оценка

$$
\frac{N_{n+p m}}{N_{n}}=\prod_{k=1}^{m} \frac{N_{n+k p}}{N_{n+(k-1) p}} \geqslant \lambda^{m}
$$

Поэтому для конечно-лакунарной последовательности (16) всегда найдется наименьшее натуральное число $q$ такое, что

$$
\frac{N_{n+q}}{N_{n}} \geqslant 2 \quad \text { при всех } n \geqslant 1 .
$$

Условие (18) эквивалентно утверждению, что для любого натурального $n$ на промежутке $[n, 2 n)$ содержится не более $q$ членов последовательности (16). Ясно, что конечно-лакунарная последовательность - это последовательность, которую можно разбить на конечное число лакунарных. Мы всегда далее считаем, что $N_{0}=0$.

Пусть теперь задана произвольная конечно-лакунарная последовательность (16), которая для некоторого натурального $l$ при всех натуральных $n$ удовлетворяет условию $N_{n+1} \leqslant 2^{l} N_{n}$. При некотором натуральном $q$ верно условие (18). Тогда для любого натурального $n$ промежуток $[n, 2 n)$ покрывается не более чем $q+1$ промежутками вида $\left(N_{k-1}, N_{k}\right]$, и, обратно, любой промежуток $\left(N_{k-1}, N_{k}\right]$ покрывается не более чем $l+1$ промежутками вида $\left(2^{n-1}, 2^{n}\right]$. Поэтому условие $(13)$, а значит и $(11)$, эквивалентно условию

$$
\max _{m=N_{n-1}, \ldots, N_{n}}\left\|_{k=N_{n-1}+1} a_{k} e^{i k x}\right\|=o(1) \quad(\text { соответственно }=O(1)) .
$$

Это означает, что последовательность, которая удовлетворяет условию (11), можно представлять себе построенной из кусков $\left\{a_{k}: k=N_{n-1}+1, \ldots, N_{n}\right\}, n=1,2, \ldots$, 
так, что выполнено условие (19). В частности, для любой ограниченной последовательности комплексных чисел $\left\{d_{n}\right\}_{n=1}^{\infty}$ и любой последовательности действительных чисел $\left\{\varphi_{n}\right\}_{n=1}^{\infty}$ из условия (11) следует, что последовательность

$$
\left.\left\{\left\{d_{n} a_{k} e^{i k \varphi_{n}}\right\}_{k=N_{n-1}+1}^{N_{n}}: n=1,2, \ldots\right\} \in \mathrm{LC} \quad \text { (соответственно } \in \mathrm{LB}\right) .
$$

Более того, это последнее условие при дополнительном предположении $\inf \left\{\left|d_{n}\right|: n \geqslant 1\right\}$ $>0$ оказывается эквивалентным условию (11).

Пусть $n_{1} \leqslant n_{2}-$ любые целые числа и $A \geqslant 1$. Будем писать, что

$$
\left\{a_{n}\right\}_{n=n_{1}}^{n_{2}} \in \operatorname{GM}(A)
$$

если выполнено либо условие

$$
\left|a_{n_{1}}\right|+\sum_{k=n_{1}}^{m-1}\left|a_{k}-a_{k+1}\right| \leqslant A\left|a_{m}\right| \quad \text { при всех } m=n_{1}, \ldots, n_{2},
$$

либо условие

$$
\left|a_{n_{2}}\right|+\sum_{k=m}^{n_{2}-1}\left|a_{k}-a_{k+1}\right| \leqslant A\left|a_{m}\right| \quad \text { при всех } m=n_{1}, \ldots, n_{2} .
$$

Основной является

ТЕОРемА 1. Пусть для последовательности комплексных чисел $\left\{a_{n}\right\}_{n=1}^{\infty}$ существуют конечно-лакунарная последовательность (16) и число $A \geqslant 1$ такие, что для каждого натурального $k$ выполнено условие

$$
\left\{a_{n}\right\}_{n=N_{k-1}+1}^{N_{k}} \in \mathrm{GM}(A) \text {. }
$$

Тогда условие (11) әквивалентно условию (15).

Для доказательства теоремы 1 нам потребуется

ЛЕмма 1. а) Если выполнено условие (20), то

$$
\left\|\sum_{n=m}^{n_{2}} a_{n} e^{i n x}\right\| \leqslant 2 A \sum_{n=m}^{n_{2}} \frac{\left|a_{n}\right|}{n_{2}-n+1} \quad \text { nри всех } m=n_{1}, \ldots, n_{2} .
$$

b) Если выполнено условие (21), то

$$
\left\|\sum_{n=n_{1}}^{m} a_{n} e^{i n x}\right\| \leqslant 2 A \sum_{n=n_{1}}^{m} \frac{\left|a_{n}\right|}{n-n_{1}+1} \quad \text { nри всех } m=n_{1}, \ldots, n_{2} .
$$

c) $\operatorname{Ecлu}\left\{a_{n}\right\}_{n=n_{1}}^{n_{2}} \in \mathrm{GM}(A)$, mo

$$
\begin{gathered}
\left\|\sum_{n=m_{1}}^{m_{2}} a_{n} e^{i n x}\right\| \leqslant 4 A\left(\sum_{n=n_{1}}^{n_{2}} \frac{\left|a_{n}\right|}{n_{2}-n+1}+\sum_{n=n_{1}}^{n_{2}} \frac{\left|a_{n}\right|}{n-n_{1}+1}\right) \\
\quad \text { nри всех } n_{1} \leqslant m_{1} \leqslant m_{2} \leqslant n_{2} .
\end{gathered}
$$


ДОКАЗАТЕЛЬСТВо. Для любого целого $q$ и натурального $s$ имеем

$$
\begin{aligned}
\left\|\sum_{j=q}^{q+s-1} e^{i j x}\right\| & =\frac{1}{\pi} \int_{0}^{\pi / s}\left|\frac{\sin (s x / 2)}{\sin (x / 2)}\right| d x+\frac{1}{\pi} \int_{\pi / s}^{\pi}\left|\frac{\sin (s x / 2)}{\sin (x / 2)}\right| d x \\
& \leqslant 1+\frac{1}{\pi} \int_{\pi / s}^{\pi} \frac{d x}{\sin (x / 2)} \leqslant 1+\ln s \leqslant 1+\sum_{j=1}^{s-1} \frac{1}{j} \leqslant 2 \sum_{j=1}^{s} \frac{1}{j} .
\end{aligned}
$$

Отсюда, если вьполнено условие $(21)$, то при $m=n_{1}, \ldots, n_{2}$ получаем

$$
\begin{aligned}
\left\|\sum_{n=n_{1}}^{m} a_{n} e^{i n x}\right\| & =\left\|\sum_{n=n_{1}}^{m-1}\left(a_{n}-a_{n+1}\right) \sum_{j=n_{1}}^{n} e^{i j x}+a_{m} \sum_{j=n_{1}}^{m} e^{i j x}\right\| \\
& \leqslant 2 \sum_{n=n_{1}}^{m-1}\left|a_{n}-a_{n+1}\right| \sum_{j=n_{1}}^{n} \frac{1}{j-n_{1}+1}+2\left|a_{m}\right| \sum_{j=n_{1}}^{m} \frac{1}{j-n_{1}+1} \\
& =2 \sum_{j=n_{1}}^{m} \frac{1}{j-n_{1}+1}\left(\sum_{n=j}^{m-1}\left|a_{n}-a_{n+1}\right|+\left|a_{m}\right|\right) .
\end{aligned}
$$

Поскольку в силу (21)

$$
\left|a_{m}\right|+\sum_{n=j}^{m-1}\left|a_{n}-a_{n+1}\right| \leqslant\left|a_{n_{2}}\right|+\sum_{n=j}^{n_{2}-1}\left|a_{n}-a_{n+1}\right| \leqslant A\left|a_{j}\right|,
$$

то имеем (24). Аналогично, из (20) и равенства

$$
\sum_{n=m}^{n_{2}} a_{n} e^{i n x}=\sum_{n=m+1}^{n_{2}}\left(a_{n}-a_{n-1}\right) \sum_{j=n}^{n_{2}} e^{i j x}+a_{m} \sum_{j=m}^{n_{2}} e^{i j x}
$$

получаем (23). Из (23) и (24) следует (25). Лемма 1 полностью доказана.

ДокАЗАТЕЛЬСТво тЕоРЕмЫ 1. По теореме А из (11) всегда вытекает (15).

Докажем обратное. Предположим, что вьполнено (15). Если $\left\{a_{n}\right\}_{n=n_{1}}^{n_{2}} \in \mathrm{GM}(A)$, то для любых $n_{1} \leqslant m_{1} \leqslant m_{2} \leqslant n_{2}$, очевидно, $\left\{a_{n}\right\}_{n=m_{1}}^{m_{2}} \in \operatorname{GM}(A)$. Поэтому, добавляя, если нужно, к последовательности $\left\{N_{k}\right\}_{k=1}^{\infty}$ точки $\left\{2^{k}-1\right\}_{k=1}^{\infty}$, можно считать, что конечно-лакунарная последовательность (16) удовлетворяет также условию $N_{k} \leqslant 2 N_{k-1}+1$ при всех $k \geqslant 1$. В силу (25) при всех натуральных $k, m_{1}$ и $m_{2}$ таких, что $N_{k-1}<m_{1} \leqslant m_{2} \leqslant N_{k}$, имеем

$$
\left\|\sum_{n=m_{1}}^{m_{2}} a_{n} e^{i n x}\right\| \leqslant 4 A\left(\sum_{n=N_{k-1}+1}^{N_{k}} \frac{\left|a_{n}\right|}{N_{k}-n+1}+\sum_{n=N_{k-1}+1}^{N_{k}} \frac{\left|a_{n}\right|}{n-N_{k-1}}\right),
$$

а последняя величина в силу условия (15) при $k$, стремящемся к бесконечности, есть $o(1)$ (соответственно $O(1)$ ). Таким образом, верно условие (19), которое эквивалентно условию (11). Теорема 1 доказана.

Из теоремы 1 вытекает 
ТЕОРема 2. Пусть для последовательности действительных чисел $\left\{a_{n}\right\}_{n=1}^{\infty}$ существуют конечно-лакунарная последовательность (16) и число $A \geqslant 1$ такие, что для каждого натурального $k$ найдутся неотрицательная неубьвающая функuия $\varphi_{k}(n)$ и неотрицательная невозрастающая функиия $\psi_{k}(n)$ и знак $s_{k}= \pm 1, \partial \Omega_{я}$ которых

$$
a_{n}=s_{k} \varphi_{k}(n) \psi_{k}(n) \quad \text { nрu всех } n=N_{k-1}+1, \ldots, N_{k}
$$

илибо

$$
\varphi_{k}\left(N_{k}\right) \leqslant A \varphi_{k}\left(N_{k-1}+1\right)
$$

либо

$$
\psi_{k}\left(N_{k-1}+1\right) \leqslant A \psi_{k}\left(N_{k}\right)
$$

Тогда условие (11) эквивалентно условию (15).

ДокАЗАТЕЛЬСтво. Возьмем любое натуральное $k$. Предположим, что имеет место (27). Тогда при любых натуральных $m=N_{k-1}+1, \ldots, N_{k}$ имеем

$$
\begin{aligned}
\left|a_{N_{k}}\right| & +\sum_{n=m}^{N_{k}-1}\left|a_{n}-a_{n+1}\right| \\
\leqslant & \varphi_{k}\left(N_{k}\right) \psi_{k}\left(N_{k}\right) \\
& +\sum_{n=m}^{N_{k}-1}\left(\varphi_{k}(n)\left(\psi_{k}(n)-\psi_{k}(n+1)\right)+\psi_{k}(n+1)\left(\varphi_{k}(n+1)-\varphi_{k}(n)\right)\right) \\
= & 2 \varphi_{k}\left(N_{k}\right) \psi_{k}\left(N_{k}\right)-\varphi_{k}(m) \psi_{k}(m)+2 \sum_{n=m}^{N_{k}-1} \varphi_{k}(n)\left(\psi_{k}(n)-\psi_{k}(n+1)\right) \\
\leqslant & 2 \varphi_{k}\left(N_{k}\right) \psi_{k}\left(N_{k}\right)+2 \varphi_{k}\left(N_{k}\right) \sum_{n=m}^{N_{k}-1}\left(\psi_{k}(n)-\psi_{k}(n+1)\right) \\
= & 2 \varphi_{k}\left(N_{k}\right) \psi_{k}(m) \leqslant 2 A\left|a_{m}\right| .
\end{aligned}
$$

Поэтому

$$
\left\{a_{n}\right\}_{n=N_{k-1}+1}^{N_{k}} \in \operatorname{GM}(2 A) .
$$

Аналогично, из (28) имеем

$$
\left|a_{N_{k-1}+1}\right|+\sum_{n=N_{k-1}+1}^{m}\left|a_{n}-a_{n+1}\right| \leqslant 2 A\left|a_{m}\right| \quad \text { при } m=N_{k-1}+1, \ldots, N_{k} .
$$

Поэтому и в этом случае верно (29). Таким образом, $(22)$ выполнено с постоянной $2 \mathrm{~A}$ вместо $A$. Осталось применить теорему 1 , и теорема 2 доказана.

Отметим, что если последовательность $\left\{a_{n}\right\}_{n=1}^{\infty}$ не убывает и неотрицательна на промежутке $n=N_{k-1}+1, \ldots, N_{k}$, то мы можем взять $\varphi_{k}(n)=a_{n}, \psi_{k}(n)=1$ на этом промежутке; условие (28) будет выполнено при $A=1$. Аналогично, если последовательность $\left\{a_{n}\right\}_{n=1}^{\infty}$ не возрастает и неотрицательна на промежутке $n=N_{k-1}+1, \ldots, N_{k}$, то можно взять $\varphi_{k}(n)=1, \psi_{k}(n)=a_{n}$ на этом промежутке; в этом случае будет вьполнено условие (27) при $A=1$. В более общем случае, ког да последовательность $\left\{a_{n}\right\}_{n=1}^{\infty}$ монотонна на промежутке $n=N_{k-1}+1, \ldots, N_{k}$ и не сохраняет знак, указанньй промежуток можно разбить на два промежутка, на каждом из которых последовательность $\left\{a_{n}\right\}_{n=1}^{\infty}$ монотонна и сохраняет знак. Поэтому из теоремы 2 сразу вытекает 
СлЕДСТВИЕ 1. Пусть для последовательности действительных чисел $\left\{a_{n}\right\}_{n=1}^{\infty}$ существует конечно-лакунарная последовательность (16) такая, что на кахсдом промежутке $n=N_{k-1}+1, \ldots, N_{k}$ последовательность $\left\{a_{n}\right\}_{n=1}^{\infty}$ монотонна, т.е. либо не возрастает, либо не убивает. Тогда условие (11) әквивалентно условию (15).

Из теоремы 2 также следует

ТЕОРемА 3. Пусть для последовательности действительных чисел $\left\{a_{n}\right\}_{n=1}^{\infty}$ существуют конечно-лакунарная последовательность (16), ограниченная последовательность действительных чисел $\left\{\theta_{k}\right\}_{k=1}^{\infty}$ и неотрицательное иелое число $m$ такие, что для каждого натурального $k$ найдется многочлен $P_{k}$ степени не выuе $m$, для которого

$$
a_{n}=P_{k}(n) n^{\theta_{k}} \quad \text { nри всех } n=N_{k-1}+1, \ldots, N_{k}
$$

Тогда условие (11) эквивалентно условию (15). Более того, если для последовательности (16) существуют полохстельные числа $\delta$ и в такие, что

$$
N_{k}-N_{k-1} \geqslant \delta N_{k}^{\varepsilon} \quad \text { nри всех } k \geqslant 1,
$$

то условие (11) әквивалентно условию

$$
a_{n} \ln n=o(1) \quad(\text { соответственно }=O(1))
$$

ДокАЗАТЕЛЬСТво. Пусть вьполнено (30). Можно считать, что $N_{k} \leqslant 2 N_{k-1}+1$ при всех $k \geqslant 1$. При каждом натуральном $k$, если многочлен $P_{k}$ не тождествен постоянной, на промежутке $\left[N_{k-1}+1, N_{k}\right]$ имеется не более $2 m-1$ точек $x$, где либо $P_{k}(x)=0$, либо производная $P_{k}^{\prime}(x)=0$. Для каждой такой точки $x$ между $N_{k-1}+1$ и $N_{k}$ вставим целое число $[x]$. Тогда между $N_{k-1}+1$ и $N_{k}$ будет вставлено не более $2 m-1$ цельх чисел. Дополним этими вставленными точками последовательность (16). Дополненная таким образом последовательность (16) также будет конечно-лакунарной. Сохраним за расширенной последовательностью прежнее обозначение $\left\{N_{n}\right\}_{n=1}^{\infty}$. Тогда на каждом промежутке $\left[N_{k-1}+1, N_{k}\right]$ соответствуюший многочлен $P_{k}$ будет монотонным и одного знака. Поскольку на этом промежутке множитель $n^{\theta_{k}}$ также монотонен и сохраняет знак, то всегда верно представление (26), где в качестве одной из функций $\varphi_{k}$ или $\psi_{k}$ можно взять тождественно равную либо 1 , либо $n^{\theta_{k}}$. Для этой функции или условие (27), или условие (28) будет выполнено. По теореме 2 получаем первую часть теоремы 3 .

Предположим теперь, что для некоторых положительных чисел $\delta$ и $\varepsilon$ выполнено условие (31). Можно считать, что $\delta, \varepsilon \in(0,1)$ и $N_{k} \leqslant 2 N_{k-1}+1$ при $k \geqslant 1$. Докажем, что тогда условие (15) эквивалентно условию (32). Действительно, из (32) всегда вытекает (15). Покажем обратное. Пусть верно условие (15). Для каждого натурального $k$ обозначим через $M_{k}$ максимум функции $\left|P_{k}(x)\right|$ на отрезке $\left[N_{k-1}+1, N_{k}\right]$. По известному неравенству Маркова

$$
\left|P_{k}^{\prime}(x)\right| \leqslant \frac{2 m^{2} M_{k}}{N_{k}-N_{k-1}-1}
$$


при всех $x \in\left[N_{k-1}+1, N_{k}\right]$. Поэтому найдутся целые $N_{k-1} \leqslant m_{1} \leqslant m_{2} \leqslant N_{k}$ такие, что $\left|P_{k}(x)\right| \geqslant M_{k} / 2$ при всех $x \in\left[m_{1}, m_{2}\right]$ и

$$
m_{2}-m_{1} \geqslant \frac{N_{k}-N_{k-1}-1}{4 m^{2}}-2 \geqslant \frac{\delta}{8 m^{2}} N_{k}^{\varepsilon}
$$

при всех достаточно больших $k$. Тогда из (15) имеем

$$
\frac{1}{2} M_{k} \sum_{n=m_{1}}^{m_{2}} \frac{n^{\theta_{k}}}{n-m_{1}+1} \leqslant \sum_{n=m_{1}}^{m_{2}} \frac{\left|a_{n}\right|}{n-m_{1}+1}=o(1) \quad(\text { соответственно }=O(1)) .
$$

Поэтому $M_{k} N_{k}^{\theta_{k}} \ln N_{k}=o(1)$ (соответственно $\left.=O(1)\right)$. Отсюда немедленно вытекает (32). Теорема 3 полностью доказана.

Хорошо известно (см. [3, гл. $1, \S 60])$, что если ряд (1) является рядом Фурье, то его частные суммы сходятся (соответственно ограничены) в метрике $L$ тогда и только тогда, когда вьполнено условие (2). Поэтому для рядов Фурье вида (10) или (12) условие (11) эквивалентно сходимости (соответственно ограниченности) частных сумм этого ряда в метрике $L$. Последнее, конечно, верно и для рядов вида (1), т.е. если ряд (1) является рядом Фурье, то его коэффициенты $\left\{c_{n}\right\}_{n=-\infty}^{+\infty}$ образуют LC-последовательность (соответственно LB-последовательность) тогда и только тогда, когда его частные суммы сходятся (соответственно ограничены) в метрике $L$. Отметим, что для проверки условия (3) достаточно проверить эквивалентное условие (8) или (9), используя, например, теоремы $1-3$.

В случае, когда последовательность $\left\{a_{n}\right\}_{n=1}^{\infty}$ квазимонотонна, т.е. неотрицательна и для некоторого $\theta>0$ последовательность $\left\{a_{n} n^{-\theta}\right\}_{n=1}^{\infty}$ не возрастает, в условии (26) можно взять $s_{k}=1, \varphi_{k}(n)=n^{\theta}, \psi_{k}(n)=a_{n} n^{-\theta}, N_{k}=2^{k}$. Тогда условие (27) выполнено. Этот один из важнейших частных случаев теоремы 2 для рядов Фурье был ранее получен Теляковским и Фоминым в работе [4] (см. также [5]).

Важнейший частный случай теоремы 3 при $m=0$, когда все $\theta_{k}=0$ и последовательность (16) лакунарна, для рядов Фурье вида (10) или (12) был ранее также получен Теляковским [6].

Отметим, что мы рассматриваем условие (2) для произвольных тригонометрических рядов, а не только рядов Фурье.

Возьмем произвольную последовательность неотрищательнох целых чисел $\left\{m_{k}\right\}_{k=1}^{\infty}$ такую, что $m_{k} \leqslant 2^{k-1}$ при всех $k \geqslant 1$. Пусть $\left\{d_{k}\right\}_{k=1}^{\infty}-$ произвольная последовательность. Положим

$$
a_{n}= \begin{cases}d_{k} & \text { при } n=2^{k-1}, \ldots, 2^{k-1}+m_{k}-1, \\ 0 & \text { при } n=2^{k-1}+m_{k}, \ldots, 2^{k-1}\end{cases}
$$

где $k=1,2, \ldots$ Тогда условие $(11)$ эквивалентно условию $(14)$, а это последнее условие, очевидно, эквивалентно условию

$$
d_{k} \ln \left(m_{k}+1\right)=o(1) \quad(\text { соответственно }=O(1)) .
$$

Возьмем произвольную последовательность положительных чисел $\left\{\varepsilon_{k}\right\}_{k=1}^{\infty}$, нижний предел которой равен нулю. Покажем, что если в $(31) \varepsilon$ заменить на $\varepsilon_{k}$, то вторая часть теоремы 3 перестанет быть верной. Действительно, полагая $N_{k}=2^{k-1}, m_{k}=N_{k}^{\varepsilon_{k}}$, 
мы видим, что условие (33) не эквивалентно условию (32) для некоторой последовательности $\left\{d_{k}\right\}_{k=1}^{\infty}$, например: $d_{k_{j}}=k_{j}{ }^{-1} \varepsilon_{k_{j}}{ }^{-1 / 2}$ для некоторой возрастающей последовательности натуральных чисел $\left\{k_{j}\right\}_{j=1}^{\infty}$ такой, что подпоследовательность $\left\{\varepsilon_{k_{j}}\right\}_{j=1}^{\infty}$ стремится к нулю, и $d_{k}=0$ для всех остальных $k$. Таким образом, при замене в (31) $\varepsilon$ на $\varepsilon_{k}$ условие (33) останется вьполненным, а условие (32) нет. Поэтому условие (31) сушественно для справедливости второй части теоремы 3 и в описанном вьше смысле неулучшаемо.

Отметим, что имеется довольно много работ (см., например, [4]-[8] и указанную там литературу), в которых для рядов Фурье при некоторых условиях на коэффициенты ряда получается эквивалентность (2) и (32).

Доказанные теоремы 1-3 показывают, что условие (15) в некоторых случаях лучше отражает поведение величины $\left\|\sigma_{n}-S_{n}\right\|$, чем условие (32). Рассмотрим несколько более подробно отношение между условиями (15) и (32).

Ясно, что из условия (32) всегда вытекает условие (15). Остановимся на некоторых условиях на последовательность $\left\{a_{n}\right\}_{n=0}^{\infty}$, при которых из (15) следует (32).

Прежде всего отметим, что из $(15)$ при $n \leqslant N \leqslant 2 n$ вытекает

$$
\begin{aligned}
\sum_{k=n}^{N}\left|a_{k}\right| \sum_{j=1}^{N-n+1} \frac{1}{j} & \leqslant \sum_{m=0}^{N-n} \sum_{k=n+m}^{N} \frac{\left|a_{k}\right|}{k-(n+m)+1}+\sum_{j=1}^{N-n} \sum_{k=n}^{N} \frac{\left|a_{k}\right|}{k-n+j+1} \\
& \leqslant \sum_{m=0}^{N-n} \sum_{k=n+m}^{2(n+m)} \frac{\left|a_{k}\right|}{k-(n+m)+1}+(N-n) \sum_{k=n}^{2 n} \frac{\left|a_{k}\right|}{k-n+1} .
\end{aligned}
$$

Поэтому из (15) всегда следует условие

$$
\frac{1+\ln (N-n+1)}{N-n} \sum_{k=n}^{N}\left|a_{k}\right|=o(1) \quad(=O(1)) \quad \text { при } n \leqslant N \leqslant 2 n .
$$

Отсюда (см. [1, доказательство теоремы 1]), в частности, вытекают условия (4) и (5) из [1]. Таким образом, рассматривая случаи $N=n+\left[n^{\varepsilon}\right], N=2 n$ и, если $\varepsilon<1$, $n=N-\left[N^{\varepsilon}\right]$, для любого $\varepsilon \in(0,1]$ из $(15)$ и $(34)$ вьводим

$$
n^{-\varepsilon} \sum_{k=n-\left[n^{\varepsilon}\right]}^{n+\left[n^{\varepsilon}\right]}\left|a_{k}\right|=o\left(\frac{1}{\ln (n+1)}\right) \quad\left(=O\left(\frac{1}{\ln (n+1)}\right)\right) .
$$

Поэтому если для некоторых $\varepsilon \in(0,1]$ и $C>0$ будет

$$
\left|a_{n}\right| \leqslant C n^{-\varepsilon} \sum_{k=n-\left[n^{\varepsilon}\right]}^{n+\left[n^{\varepsilon}\right]}\left|a_{k}\right| \quad \text { при всех } n \geqslant 1,
$$

то из (15) следует условие (35), а значит, и (32).

Поскольку при $\varepsilon \in(0,1]$ имеем

$$
\begin{aligned}
\left|a_{n}\right| \sum_{k=n}^{n+\left[n^{\varepsilon}\right]} \frac{1}{k-n+1} & =\sum_{k=n}^{n+\left[n^{\varepsilon}\right]} \frac{\left|a_{k}\right|}{k-n+1}+\sum_{k=n}^{n+\left[n^{\varepsilon}\right]} \frac{\left|a_{n}\right|-\left|a_{k}\right|}{k-n+1} \\
& \leqslant \sum_{k=n}^{n+\left[n^{\varepsilon}\right]} \frac{\left|a_{k}\right|}{k-n+1}+\max _{k=n, \ldots, n+\left[n^{\varepsilon}\right]}|| a_{n}|-| a_{k}|| \sum_{k=n}^{n+\left[n^{\varepsilon}\right]} \frac{1}{k-n+1},
\end{aligned}
$$


то из (15) и условия

$$
\max _{k=n, \ldots, n+\left[n^{\varepsilon}\right]}|| a_{n}|-| a_{k}||=o\left(\frac{1}{\ln (n+1)}\right) \quad\left(=O\left(\frac{1}{\ln (n+1)}\right)\right)
$$

вытекает (32). Таким образом, если последовательность $\left\{a_{n}\right\}_{n=1}^{\infty}$ такова, что при некотором $\varepsilon \in(0,1]$ верно $(36)$ или $(37)$, то условия $(15)$ и (32) эквивалентны. В частности, в этом случае из (11) вытекает (32).

ЗАмЕЧАнИЕ 1. Если в линейном пространстве всех LB-последовательностей ввести норму по формуле

$$
\left\|\left\{c_{n}\right\}_{n=-\infty}^{\infty}\right\|_{\mathrm{LB}}=\sup _{n \geqslant 0} \max _{n \leqslant m \leqslant 2 n}\left\|S_{m}-S_{n-1}\right\|
$$

где $S_{n}$ - частные суммы ряда $(1), S_{-1}=0$, то получим банахово пространство LB. Тогда линейное пространство всех LC-последовательностей является замкнутым подпространством в банаховом пространстве LB, т.е. также является банаховым пространством с той же самой нормой.

Отметим также, что если $\left\{c_{-n}\right\}_{n=-\infty}^{+\infty} \in \mathrm{LC}$ (соответственно $\in \mathrm{LB}$ ), то из (13) следует, что последовательности $\left\{c_{n+1}\right\}_{n=-\infty}^{+\infty},\left\{c_{n-1}\right\}_{n=-\infty}^{+\infty} \in \mathrm{LC}$ (соответственно $\left.\in \mathrm{LB}\right)$ и для любого натурального $m$ и целого $j$ последовательность $\left\{c_{m k+j}\right\}_{k=-\infty}^{\infty} \in \mathrm{LC}$ (соответственно $\in$ LB). В частности,

$$
\left\{c_{n}\right\}_{n=-\infty}^{+\infty} \in \mathrm{LC} \quad(\in \mathrm{LB}) \Longleftrightarrow(\forall j=0, \ldots, m-1)\left\{c_{m k+j}\right\}_{k=-\infty}^{\infty} \in \mathrm{LC} \quad(\in \mathrm{LB}),
$$

т.е. для любого натурального $m$ условие (11) эквивалентно условию

$$
(\forall j=0, \ldots, m-1)\left\{a_{m k+j}\right\}_{k=1}^{\infty} \in \mathrm{LC} \quad(\text { соответственно } \in \mathrm{LB}) .
$$

\section{СПИСОК ЦИТИРОВАННОЙ ЛИТЕРАТУРЫ}

[1] Белов А. С. Об условиях сходимости в среднем тригонометрических рядов Фурье // Изв. Тульского гос. университета. Сер. матем., мех., информатика. 1998. Т. 4. №1. С. 40-46.

[2] Белов А. С. Об условиях сходимости (ограниченности) в среднем частных сумм тригонометрического ряда // Сб. статей "Метрическая теория функций и смежные вопросы анализа". М.: АФЦ, 1999. С. 1-17.

[3] Бари Н. К. Тригонометрические ряды. М.: Физматгиз, 1961.

[4] Теляковский С. А., Фомин Г. А. О сходимости в метрике $L$ рядов Фурье с квазимонотонньми коэффициентами // Тр. МИАН. 1975. Т. 134. С. 310-313.

[5] Garret J.W., Rees C.S., Stanojević Č. V. On $L^{1}$-convergence of Fourier series with quasi-monotone coefficients // Proc. Amer. Math. Soc. 1978. V. 72. № 3. P. 535-538.

[6] Теляковский С. А. О сходимости в метрике $L$ тригонометрических рядов с редко меняющимися коэффициентами // Тр. МИАН СССР. 1991. Т. 200. С. 322-326.

[7] Фомин Г. А. О сходимости рядов Фурье в среднем // Матем. сб. 1979. Т. 110. № 2. С. 251-265.

[8] Fridli S. On the $L^{1}$-convergence of Fourier series // Studia. Math. 1997. V. 125. № 2. P. 161-174. 